EOMmun Communication et organisation

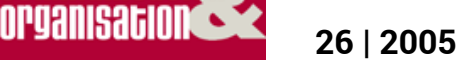

La communication des nouvelles éthiques de l'entreprise

\title{
La communication politique
}

De Jacques Gerstlé, Paris, Armand Colin, Compact Civis, Paris, 2004

\section{Kuzmanovic Djordje}

\section{OpenEdition}

Journals

Édition électronique

URL : http://journals.openedition.org/communicationorganisation/3305

DOI : 10.4000/communicationorganisation.3305

ISSN : $1775-3546$

Éditeur

Presses universitaires de Bordeaux

Édition imprimée

Date de publication : 2 janvier 2005

Pagination : 263-270

ISSN : 1168-5549

Référence électronique

Kuzmanovic Djordje, «La communication politique », Communication et organisation [En ligne], 26 |

2005, mis en ligne le 19 juin 2012, consulté le 21 septembre 2020. URL : http://

journals.openedition.org/communicationorganisation/3305; DOI : https://doi.org/10.4000/

communicationorganisation.3305

Ce document a été généré automatiquement le 21 septembre 2020.

(C) Presses universitaires de Bordeaux 


\title{
La communication politique
}

\author{
De Jacques Gerstlé, Paris, Armand Colin, Compact Civis, Paris, 2004
}

\section{Kuzmanovic Djordje}

\section{NOTE DE L'AUTEUR}

Jacques Gerstlé est professeur au département de science politique de l'Université Paris 1 (Panthéon-Sorbonne), membre du Centre de Recherche Politique de la Sorbonne (Umr-CNRS). Il est responsable du Diplôme d'Etudes Supérieures Spécialisées en Communication Politique et Sociale.

1 La communication politique peut être abordée sous un angle tant théorique que pratique. Le plus souvent, les auteurs qui traitent de cette discipline ne s'intéressent qu'à un seul de ces deux aspects et généralement en ne se penchant que sur un nombre restreint de concepts ou de pratiques. Tout au contraire, dans son livre, La communication politique, Jacques Gerstlé dresse un tableau de synthèse des connaissances accumulées dans le domaine. De fait, un des grands mérite de l'ouvrage est de permettre aux lecteurs d'opérer un croisement immédiat entre les différents paradigmes de la communication politique, de confronter les concepts; en somme, il rend possible un "dialogue" des idées les unes avec les autres - démarche particulièrement heuristique, tant pour le spécialiste que pour le citoyen. Loin de faire uniquement un recensement, l'auteur mène une réflexion poussée, dont il ressort une idée force, à savoir, la prédominance du politique dans les pratiques de communication politique. Ainsi, dans un développement allant du théorique au pratique, Jacques Gerstlé montre que même si la communication politique se présente comme un ensemble hétéroclite de théories et de techniques, elle est également, et peut-être surtout, inspiratrice de stratégies et de conduites variables selon les positions de pouvoir occupées et les situations concrètes vécues par les acteurs de la vie politique. L'auteur s'est donné comme projet d'intégrer les aspects multiples de la communication, trop souvent artificiellement dissociés. Pour réaliser ce projet ambitieux, l'ouvrage est divisé en deux parties distinctes. 
2 La première partie consiste en un recensement exhaustif des diverses théories de la communication politique et les dimensions qui les caractérisent tant à l'étranger qu'en France. L'auteur y présente également la modernisation technique des arènes du jeu politique, tout en menant une réflexion originale sur les effets de la communication politique, notamment en montrant qu'il est permis de considérer qu'il existe tant des effets de communication persuasive que des mécanismes persuasifs de l'information. La souplesse conceptuelle qui caractérise les notions de communication et de politique ne rend pas aisée leur enfermement dans des définitions étanches. L'auteur, très humblement, ne s'y risque pas, préférant montrer la polysémie de la communication politique en présentant quatre conceptions de cette discipline (instrumentale, œcuménique, compétitive et délibérative), mais attribue une centralité au phénomène de la communication dans l'organisation et le fonctionnement de la société. Selon Jacques Gerstlé, loin de produire un phénomène isolable, politique et communication s'articulent de façon diffuse et sont largement consubstantielle : la res publica ne serait pas possible sans la communication pour remédier à l'indétermination du politique. Par ailleurs, les apports de la science empirique de la communication à la compréhension de certains aspects de la communication politique sont mis en lumière et mis en relation avec les théories pures, ce qui est relativement original dans ce type d'ouvrage et particulièrement stimulant, surtout pour les professionnels de la discipline.

3 L'évolution de l'espace public politique est envisagé ici sous quatre angles principaux : la médiatisation de la vie politique, les techniques de rationalisation de la vie politique (en particulier le marketing politique), les sondages et les études quantitatives et enfin la publicité, observée par le biais des menaces qu'elle fait peser sur l'espace public. Ici l'auteur nous invite à remonter le temps en faisant une histoire de ces évolutions et en montrant comment la dimension technique tend à induire de nouvelles pratiques dans le cadre d'une société de communication, issue de la révolution des médias. Une attention particulière est portée sur le développement des moyens audiovisuels de communication de masse, changeant les modalités de fonctionnement de la communication politique et dont découle l'adoption de nouvelles technologies politiques. Bien qu'il souligne l'importance des mutations techniques, Jacques Gerstlé montre que, de la rhétorique de l'antiquité grecque à la télévision du $\mathrm{XX}^{\mathrm{e}}$ siècle, la politique a toujours présenté une dimension technique, et que par voie de conséquence, son appréhension par ce seul aspect fait perdre de vue sa substance réelle. Si l'on peut se permettre un parallèle avec le concept de la guerre, cela revient à s'interroger sur ce dernier comme Jacques Gerstlé s'interroge sur la politique : de l'épée de bronze à l'arme nucléaire, la guerre a certes changé de visage, mais a-t-elle changé d'âme?

Si la communication politique est une façon pacifiée de régler les conflits, elle est également stratégie symbolique de domination; dans les deux cas, elle suppose le recours à la persuasion, entendue comme un processus de communication dont l'objectif est un déplacement des opinions sur lesquelles se fondent les prises de décision. Dès lors, la communication politique, plus que simple agrégation des préférences dans le cadre des affrontements électoraux, en permet la transformation. Jacques Gerstlé analyse en profondeur cette face potentiellement - mais pas nécessairement - déceptive de la communication politique, en examinant les données d'enquêtes accumulées depuis une dizaine d'années sur la convergence des résultats attribués à la communication politique en terme de changement d'attitude. Il distingue la communication contrôlée dont l'objet est de produire un effet direct de persuasion et l'information diffusée par 
les médias qui engendre des effets de persuasion indirecte. Résolument, l'auteur s'oppose à une vision manichéenne où l'information alimenterait la conviction et où la persuasion serait sensée nourrir la manipulation. Quatre procédés de persuasion sont ici mis en lumière : la persuasion directe (qui a pour objectif l'ajout d'informations auprès des récepteurs pour étayer leurs croyances), les effets de cadrage (qui activent des conceptions déjà présentes afin d'en modifier l'importance), les effets d'amorçage (rendant certaines considérations momentanément plus accessibles) et les effets d'agenda. Ces derniers agissent aussi sur les mécanismes des variations d'accessibilité de l'information, mais Jacques Gerstlé préfère y voir, non pas une forme de manipulation des citoyens, mais plutôt une pression exercée sur leur attention. Tout en considérant que l'information est destinée à produire des effets prioritairement cognitifs et en abordant les relations des effets à la réception, l'auteur distingue nettement les processus de persuasion stricto sensu et les mécanismes persuasifs de l'information pour conclure que leur concomitance facilite l'emprise sur l'attention et les perceptions publiques.

Dans la deuxième partie de l'ouvrage, Jacques Gerstlé montre comment les diverses positions de pouvoir sont en rapport étroit avec l'usage - conjoint et disjoint - de l'information et de la communication, avec cette particularité de ne jamais dissocier l'une de l'autre. Cette analyse est tout d'abord considérée sous l'angle des problèmes posés par la conquête des postes électifs, en mettant en avant l'importance de cette dernière comme objet d'analyse fortement centré sur la variable de la communication, puis sous l'angle des problèmes mis à jours par l'exercice du pouvoir, alors qu'il y a une recherche, de la part des gouvernants, du soutien populaire le plus large possible.

6 L'intérêt principal de cette seconde partie tient non seulement dans son illustration de la première, mais également dans le fait qu'elle donne vie aux théories précédemment exposées, laissant ainsi le loisir aux lecteurs de se faire leurs propres opinions sur la validité opérationnelle des différents paradigmes en constatant les effets concrets de la communication politique. Jacques Gerstlé fait là un effort de dévoilements des images dansantes sur le fond de la grotte et interroge le citoyen qui sommeil dans le lecteur.

Dans la première partie l'auteur a montré que la communication politique était marquée par une ambiguïté conceptuelle, renforcée par l'affrontement de théories et l'ubiquité des phénomènes. Pour palier cette difficulté de dire l'objet, il y a ici un parti pris fort de cerner les rapports qu'entretient la communication avec la politique par l'analyse de ses pratiques. Par ailleurs, en soulignant l'importance des flux de communication transnationaux, une réflexion est menée sur l'émergence d'une hypothétique opinion publique internationale. Ainsi, la possibilité ouverte par les propriétés du système international de production et de diffusion de l'information de masse incite à comprendre la possibilité stratégique de la désinformation, entendue comme manipulation de l'information réputée objective, notamment dans les phases de crise. C'est toutefois l'impact de la communication dans l'ordre politique interne qui est ici particulièrement développé, en examinant les pratiques à partir des positions de pouvoir, conduisant à distinguer entre les contributions de la communication à la conquête du pouvoir, à son exercice et à la participation des citoyens grâce à de nouvelles formes d'engagement civique.

8 Tout en soulignant que les élections constituent un moment fort de la vie politique, l'auteur insiste sur le fait qu'elles ne sauraient épuiser toutes les activités de communication politique. A contrario, le pouvoir conquis doit être conservé et la 
tendance croissante à transformer la vie politique en campagnes permanentes incite à penser que la communication devient un aspect important de cet exercice. Pour étudier ce phénomène, la communication est analysée au regard de la position occupée, d'abord, par le Président de la République, puis par gouvernement et enfin par l'étude des facteurs de la popularité.

9 Après un aperçu des différentes théories de la démocratie et du degré qu'elles autorisent à la participation politique, l'auteur fait le constat qu'en tout état de cause, la communication est une condition de la participation. Il remarque que l'accès à la communication pour le plus grand nombre, ne semble pas être une condition suffisante à la participation politique. Ainsi est globalement critiquée l'hypothèse optimiste selon laquelle l'opulence communicationnelle induirait automatiquement une expansion de la démocratie. Pour comprendre la participation des citoyens à la vie politique, l'auteur intègre les pratiques non conventionnelles de ceux-ci, ainsi que la communication plus intense des citoyens qui se tiennent politiquement informés et qui envoient des messages aux journalistes et aux leaders politiques. Ainsi, l'auteur appréhende les représentations et pratiques en matière d'information et de communication, les modes d'expression et d'action collective des citoyens (la démocratie participative, les transformations de la démocratie locale ou les nouvelles pratiques délibératives), pour montrer qu'en termes macrosociologiques, la participation politique est bien un processus de révélation tant des préférences que des modes de transmission au système politique. Jacques Gerstlé termine ainsi son livre en menant une réflexion approfondie sur les différents défis que la démocratie pose à l'information et à la communication.

10 Au total, Jacques Gerstlé montre bien que l'analyse de la communication politique se heurte à divers obstacles; ils sont d'ordre théorique, technique et pratique et ne sauraient être limitée à l'un d'entre eux comme la communication politique ne saurait être réduite à l'une de ses dimensions constitutives, le symbolique, le structurel et le pragmatique. Concernant les problèmes théoriques, l'auteur note que la tentation est grande chez les experts ou les professionnels concernés de s'emparer du concept et de chercher à en imposer une définition. Or en politique, nombreux sont les acteurs intéressés par la communication non plus sous son caractère privé d'exercice d'une profession mais sous son caractère public de révélation et de règlement des problèmes collectifs. Deuxièmement, il met en lumière une tendance moderne à mettre l'accent sur une conception technique de la communication dominée par l'idée de transfert d'information au détriment de la signification et de l'interprétation. Or, selon Jacques Gerstlé, il n'est pas évident que la logique de la communication s'impose à la logique politique.

11 Un troisième problème est posé par les discours médiacentriques qui considèrent que la politique gravite essentiellement autour de ce nouveau pouvoir. L'auteur ne met pas de côté les bouleversements technologiques et leur impact social : dans nos sociétés postindustrielles, la distance sociale peut s'accroître dans la mesure où les relations fondées sur la proximité et l'interconnaissance sont concurrencées par des relations interposées grâce aux médias qui affranchissent des contraintes de temps et d'espace. Mais, la communication est devenue une ressource - inégalement distribuée parmi les acteurs politiques - dont les médias ont accru le caractère stratégique par la diffusion massive de l'information. La communication politique agit dans un univers de différences et d'inégalités que le discours médiacentrique tend à ignorer. Ainsi, les positions de pouvoir conditionnent largement la détention des ressources de 
communication et l'élaboration des stratégies, d'autant plus que la communication politique reste fortement dépendante de contraintes économiques et que les préconstruits culturels, les codes symboliques, les normes et les règles du jeu de la communauté concernée imposent lourdement leurs contraintes.

12 Ces différents obstacles présentent le point commun de toujours rabattre le politique sur le communicationnel et donc de réduire le politique à une manifestation parmi d'autres au lieu d'y voir une forme élémentaire de la vie en société.

13 Enfin, Jacques Gerstlé insiste sur trois aspects de l'évolution de la communication politique qui lui paraissent centraux. Tout d'abord et sans nier le reflux du politique, que ce que rapportent les médias de l'acteur politique devient plus important que ce que dit l'acteur politique; c'est-à-dire que tendanciellement un plus grand crédit est donné aux médias plutôt qu'aux professionnels de la politique. Ensuite, que la référence prend valeur d'inférence: pour les citoyens ordinaires, véritables "avares cognitifs", les schématisations mènent rapidement aux conclusions dans l'évaluation des acteurs politiques et de leurs performances. Enfin et surtout, que la disjonction entre l'information et la communication contrôlée par l'acteur politique est aussi coûteuse pour lui qu'est profitable leur conjonction. Pour Jacques Gerstlé, de la délocution à la perlocution, de la référence à l'inférence, de la disjonction à la conjonction constituent trois systèmes de tensions qui sont représentatifs de ce qu'est aujourd'hui devenue la communication politique.

14 Par ailleurs, tout au long de l'ouvrage, Jacques Gerstlé essaye de répondre par petites touches à la question de savoir s'il existe une corrélation négative entre, d'une part, crise de la représentation politique, et explosion de la communication, d'autre part. Tout en mesure, bien que ses remarques tendent à valider cette position, il n'apporte pas de réponses définitives, considérant à juste titre que le recul historique manque pour répondre pleinement à cette question. Une fois encore, l'auteur prend le lecteur au sérieux, voire le responsabilise par rapport à son rôle, même minime, dans la Cité en l'invitant à se faire sa propre opinion. Soulignons enfin l'importance accordée dans cet ouvrage à l'analyse empirique, laquelle est largement pourvue en tableaux, schémas et figures et notons l'excellente indexation des notions, figures et tableaux qui rendent la lecture et la "navigation" dans le livre particulièrement agréable et aisée.

La communication politique de Jacques Gerstlé se pose donc comme un ouvrage de référence pour tous ceux qui sont intéressés de près ou de loin à cette discipline. Rédigé dans un style clair, il se prête à des lectures différenciées. Ce livre est non seulement destiné aux spécialistes scientifiques en la matière - chercheurs, enseignants ou étudiants - qui pourront se confronter aux théories et analyses exposées, mais également aux praticiens de la communication politique ou de l'information journalistes, hommes politiques, communicants professionnels - qui y trouveront de quoi alimenter une attitude réflexive sur leurs propres pratiques, ou encore aux citoyens qui refermant les dernières pages auront le sentiment qu'un voile s'est levé devant leurs yeux. 